\title{
http://dx.doi.org/10.21611/qirt.1996.061 Statistical analysis techniques for aerial infrared
images in wavelets transform domain
}

\author{
by D. Milovanovic ${ }^{1}$, B.Wiecek², A. Marincic ${ }^{1}$, Z. Barbaric ${ }^{1}$ and G. Petrovic ${ }^{1}$ \\ ${ }^{\dagger}$ Faculty of Electrical Engineering, P.O.Box 816, 11001 Belgrade. Yugoslavia \\ ${ }^{2}$ Technical University of Lodz, Institute of Electronics, Stefanowskiego 18/22, 90-924, Poland
}

\begin{abstract}
Statistical analysis of airborne IRLS (InfraRed Line-Scanner) images in DWT (Discrete Wavelet Transform) numerical domain is an extension of statistical measurements of computer generated images in our earlier works aimed to finding method for IRLS image coding. The initial results obtained with test images indicate that proposed statistical techniques in the wavelet transform domain are efficient for joint space-frequency 2D anisotropy analysis and adaptive data compression of IRLS images.
\end{abstract}

\section{Introduction}

Image analysis techniques can be classified as phenomenological, structural, deterministic, and statistic. Statistical parameters of image and corresponding radiance and terrain temperature distribution describe basic characteristics of scenes which are of practical importance when modeling scenes, evaluating and predicting IR system performance, developing algorithms for digital processing and data compression for storing or transmission. The statistical properties of high-resolution InfraRed Line-Scanner (IRLS) images of natural terrain in high demand surveillance application are analyzed in this paper.

IRLS is a mechanic-optoelectronic device for high-resolution image acquisition in the thermal IR spectral range. The terrain is sampled by a transverse scan as the aircraft moves forward along a track. An obtained series of signals, each corresponding to a terrain scanning line, forms a two dimensional image without temporal information, because each line consists of new data. Consequently, IRLS operates as a linear angle scanning system, giving angular scan and linear displacement along track as the two dimensions of the image. IRLS is more sensitive along the scan direction, giving highly correlated images with the serious geometrical distortion in approaching the horizon $[1,2]$.

Over the past years many classical statistical techniques, in space or frequency domain, which involve probability density function ( $p d f)$, autocorrelation function (acf) and power spectral density ( $\rho s d)$, have been utilized to analyze linear scan patterns in one dimension (1D). However, IRLS requires the analysis of two-dimensional (2D) aspect of acquired data. Many of the analysis techniques employed for 10 data may be either used directly by averaging over two dimension data or by generalization to a $2 \mathrm{D}$ equivalent $[3,4]$.

Three major obstacles in statistical analysis of IRLS images can be identified: nonstationarity, anisotropy and edge sensitivity. An assumption on stationarity of image background, i.e. that the statistical properties do not vary over an image, may be true for a homogenous terrain, but it is rarely valid for real IRLS images, when the image segmentation and isotropy analysis are necessary. The edges in an IRLS image carry very important spatial information about the position and size of objects. In spite of that, there is no good classical measure for the edge sensitivity of an image.

One new technique for the analysis of nonstationary images is DWT (Discrete Wavelet Transform). The DWT offers good edge localization in spatial domain and at the same time good resolution at low frequencies in the transform domain [5], which are desirable properties when analyzing highly correlated IRLS images. Also, the DWT is capable of eliminating the redundant information and providing a compact, multiresolution representation of images for efficient entropy reduction and coding.

In this paper, we propose a new application of DWT in the segmentation, edge sensitivity and anisotropy analysis. Various types of IRLS images, carefully segmented along the scan 
direction, based on our previous analyses $[1,2]$ are decomposed by 2D separable DWT filters with optimal space-frequency localization (figure 1). Our analysis is based on the comparison of histogram shape and width, variances and entropies of horizontal and vertical subimages versus decomposition level.

In the first part of the paper, classical statistical parameters are explained. Next, the DWT decomposition technique is defined and the edge sensitivity and anisotropy analysis, based on that method, is proposed. The obtained statistical results of IRLS test images are discussed in the final part of the paper.

\section{Analysis techniques}

The image is treated as an arranged $2 \mathrm{D}$ set of finite dimensions $\mid x \mathrm{~J}$, whose discrete elements (pixels) $S_{i, j}, i=1,2,3, \ldots, I, j=1,2,3, \ldots, J$ take discrete values $S=0,1,2, \ldots, 2^{B}-1$. Here $B$ denotes the number of bits used for quantizing of each pixel. Two types of test images (512x512 pixels), acquired during night from low altitude flights at 1500/3000 feet, are used in our experiments: one is a 'city' area full of details of various size and contrast, and the other one is 'fields' representing uniform-like terrain. Two analyzing segments $(256 \times 256$ and $128 \times 128$ pixels with $2^{8}$ gray levels) along scan direction of each image type are selected and analyzed (figure 2a). The first segment covers nadir and the second covers region from nadir towards the horizon.

Scaled $p d f$ of analyzed test images - histograms (figure $2 b$ ) are calculated as a relative number of appearance of every permissible pixel value

$$
p_{S}=\frac{n_{S}}{I J}
$$

The variance of image is the mean value of squared difference between pixels and mean value $m$

$$
\sigma^{2}=\frac{1}{I J} \sum_{i=1}^{I} \sum_{j=1}^{J}\left(S_{i, j}-m\right)^{2}
$$

where $m$ is the spatial mean value of all pixels $m=\frac{1}{I J} \sum_{i=1}^{I} \sum_{j=1}^{J} S_{i, j}$.

The spatial autocorrelation function $\operatorname{acf}(k)$ quantifies, on average, how values of pixels are similar and predictable as a function of their inter-pixel separation $k$. According to the WienerKihtchine relation acf and psd of a stationary process form a Fourier transform pair. However, in non stationary image analysis there are many problems in applying the Fourier transform technique: windowing, averaging, spatial-frequency resolution, aliasing and anisotropy estimation $[3,4]$.

In contrast to the Fourier transform, the Discrete Wavelet Transform offers many advantages: compact, stable multiresolution image representation, adapted to nonstationary images with good edge localization and additional spatial-oriented selectivity, as well as existence of fast computational algorithm ( $\mathrm{L}, \mathrm{H}$ filter banks) [5]. Elementary functions that form the wavelet transform base are obtained by dilation and translation of one special prototype function $\psi(x)$, called the mother wavelets

$$
\psi_{j, k}(x)=2^{-\frac{j}{2}} \psi\left(2^{-j} x-k\right)(j, k) \in Z^{2}
$$

where $j, k$ are dilation and translation indices, respectively. It should be proved that DWT is equivalent to filtering and decimating of a filtered signal. The filter bank, with perfect reconstruction, consists of a lowpass filter $L$ and highpass filter $H$, whose impulse responses are in special relationship with a mother wavelet function. In order to compute separable 2D DWT of an image, 1D FIR (Finite Impulse Response) filters are used and combined with decimating in both horizontal and vertical dimensions to compute at each level: 1) a lowpass approximation image, 2) a horizontal detail image, 3) a vertical detail image, and 4) a diagonal detail image 
(figure 1a). In a multilevel decomposition, the coarse resolution approximation image is similarly decomposed, and so on. Subimages in the two level decomposition $(j=1,2)$ DWT (FIR filters are 8-taps Binomial with optimal spatial-frequency resolution) of the second type IRLS test images are shown in figure $1 b$.

\subsection{Edge sensitivity analysis}

The spatial difference exceedance (sde) probability density function, sensitive to edges, is usually used for edge sensitivity analysis [3]. The spatial differencepdf is formed for a particular separation by differencing the intensity values of two pixels in both orthogonal directions. The degree of deviation from a Gaussian distribution of the spatial differencepdf is a measure of the edge sensitivity of an image.

In the case of DWT image decomposition, as proposed in this work, the histograms of edge subimage can be modeled with the so-called generalized Gaussian function [5], regardless of the types of test image histograms (figure $2 b$ ). In our analysis we obtained the histograms edge subimage (figure $2 c, d$ ) which seem to be between Gaussian and Laplacian pdf. The degree of deviation from the Gaussian distribution were used as a measure of the edge sensitivity of IRLS images. It is interesting to note that in a special case, when DWT is equal to Haar transform, the histograms of the edge subimages are identical to the sde probability density function formed by differencing neighbouring pixels.

\subsection{Structure function}

The structure function $D(k)$ is another statistical measure [3] which involves the mean square of the difference between pixel values for a given separation $k$ and $m=0$

$$
D(k)=\frac{1}{I} \sum_{i=1}^{1}\left(S_{i}-S_{i+k}\right)^{2}=2\left(\sigma^{2}-a c f(k)\right)
$$

In figure $3 a, b$ the evaluations of IRLS $D(k)$ in horizontal and vertical DWT subimages are shown. One can see that for IRLS images the exponential model of the correlation is initially a good approximation, as it was known to be valid for original visual images [3]. It seems to us that the exponential model is better in the DWT domain.

Generally, correlation properties of images are of special interest when developing image compression techniques. If the similarity between neighbouring pixels is large, it is possible to estimate the value of a current pixel from the values of one or more of its neighbours that have been previously coded. The normalized covariance coefficient, used in this work is given by

$$
\rho\left(k_{h}, k_{v}\right)=\frac{1}{I J} \sum_{i=1}^{I-k_{h}} \sum_{j=1}^{J-k_{v}} \frac{\left(S_{i, j}-m\right)\left(S_{i+k_{h}, j+k_{\mathrm{r}}}-m\right)}{\sigma^{2}}
$$

where $k_{h}, k_{v}=0, \pm 1, \pm 2, \ldots$ denote correlation distance in the two orthogonal directions (horizontal and vertical). The covariance coefficients between neighbouring pixels in horizontal subimages $\rho_{h}^{l}=\rho(1,0)$ versus DWT decomposition levels, are shown in figure $3 c$.

\subsection{Entropy}

The entropy value is a measure of information content of an image and at the same time determines the smallest possible bit rate in independent coding of each pixel without losses. The first order entropy is equal to the mean value of information content per one pixel [1]

$$
H=-\sum_{S=0}^{2^{B}-1} p_{S} \log _{2}\left(p_{S}\right)
$$

The first order entropy of horizontal DWT subimages $H_{h}$ of IRLS test images versus decomposition level are shown in figure $4 a$. 


\subsection{Isotropy analysis}

Subjective estimates of isotropy and anisotropy of images are very complicated, thus the assumption of isotropy of the background is inherent in developing many algorithms for data compression. A classical relative isotropy function (rif) is the ratio of the psd components in the two orthogonal directions in an image [3]. With this function it is possible to measure the isotropy of an image in a particular spatial frequency range. In this paper, we propose the calculation of rif $\left(\sigma_{h}^{2} / \sigma_{v}^{2}\right)$ as the variances ratio of horizontal and vertical subimages obtained by DWT decomposition of IRLS test images (figure $2 f$ ) versus the decomposition level. The additional advantage of DWT technique is the possibility of simultaneous anisotropy analysis in the spatial domain by comparing the edge sensitivities of horizontal and vertical subimages, as explained in 2.1. We also propose the generalization of the rif function in the anisotropy analysis of normalized covariance coefficient rif $\left(\rho_{h} / \rho_{v}\right)$ and the first-order entropy rif $\left(H_{h} / H_{v}\right)$ in horizontal and vertical subimages of IRLS test images versus the decomposition level (figure $3 d$, figure $4 b$ ).

\section{Results}

In order to analyze 2D aspect of IRLS data, we at first had to segment the images in several analysis frames. The IRLS test images of two types of terrain are subdivided into two frames: the first one covers nadir, while the second one covers the area towards the horizon, where the influence of the line scanner is considerable. Over each frame the images are assumed to be stationary: the mean value, variance, covariance coefficients and first order entropy of the first type (city area) are $\left(m=165.26, \sigma^{2}=1128.29, \rho_{h}=0.822, \rho_{v}=0.826, H=6.98\right)$, ( $\left.m=137.63, \sigma^{2}=920.52, \rho_{h}=0.851, \rho_{v}=0.895, H=6.81\right)$; while for the second type (fields) are $\left(m=153.88, \quad \sigma^{2}=594.38, \rho_{h}=0.939, \quad \rho_{v}=0.846, \quad H=6.50\right), \quad\left(m=129.60, \quad \sigma^{2}=811.11, \quad \rho_{h}=0.818\right.$, $\left.\rho_{v}=0.917, H=6.69\right)$, of frames in nadir and towards horizon, respectively.

The DWT removes much of the correlation between the neighbouring pixels in IRLS analyzed images, so that the obtained multiresolution structure is more suitable for efficient redundancy reduction and coding. Decorrelation results in concentration of subimages histograms around zero +128 , and therefore in reduced variance and entropy. The obtained statistical parameters agree with theoretical predictions: more correlated second type of images has more narrow histogram of subimages (figure $1 c, d$ ) and consequently lower variance and entropy than the first type of subimages. The identical trend of the variance and first order entropy can be noted when comparing frames 2 . and 1 . for both types of images. The degree of the anisotropy variance is also smaller for the frame 2., and is simultaneously slower then 1 , while the rif of entropy is closer to 1 and it slowly varies with the decomposition level. Those results can be used in setting of an appropriate quantizer in the efficient coding of subimages.

We found that the correlation after DWT is rested only among neighbouring pixels $\dot{k}=1,2$ in horizontal direction in horizontal oriented subimages, i.e in vertical direction in vertical oriented subimages. The important result is that the values of covariance coefficients in vertical direction (along track) are larger in the 2. frame then in 1. frame, where is also the influence of a line scanner more considerable. This disproportion becomes larger when a decomposition level increases, regardless on the type of images. These results point out on the possible adaptive predictive coding of subimages.

\section{Conclusion}

In using the DWT image decomposition, we found that it has advantages over the statistical techniques separately dedicated for the spatial and frequency domain. Applying the DWT over IRLS test images, we obtained multiresolution subimages in 4 levels with good edge localization and spatial-oriented selectivity. The statistical parameters analysis of these subimages is more meaningful than that of the original test images. DWT also improves the edge sensitivity and anisotropy analyses in along two directions, horizontal (across scan) and vertical (along track). However, further work is needed to quantify more precisely the observed anisotropy of DWT decomposed IRLS images and for their exploitation by some efficient adaptive image coding. 


\section{REFERENCES}

[1] Z.Barbaric, A.Marincic, G.Petrovic and D.Milovanovic. - Thermal images generated by a line-scanning technique: Statistical properties. Applied Optics, Vol.33 No.20, 1994.

[2] D.Milovanovic, A.Marincic, Z.Barbaric and G.Petrovic. - Statistical analysis of computer generated thermal images based on overall modeling of line-scanning process. In Proceedings QUIRT 1994, Italy, pp.13-18.

[3] E.M.Winter and M.C.Smith. - Analysis techniques for two-dimensional infrared data. In Proceedings SPIE, Vol.156, 1978, pp.20-29.

[4] N. Ben-Yosef, K. Wilner, S.Simhony and G.Feigin. - Measurement and analysis of 2-D infrared natural background. Applied Optics, Vol.24 No.14. pp.2109-2113, 1985.

[5] S.G.Mallat. - A theory for multiresolution signal decomposition: The wavelet representation. IEEE Transactions on PAMl, Vol.11, No.7, 1989.

\begin{tabular}{|c|c|c|}
\hline $\begin{array}{c}i=1 \\
j=2(\mathrm{LL})\end{array}$ & $\begin{array}{c}i=2 \\
j=2(\mathrm{LH})\end{array}$ & $\begin{array}{l}j=5 \\
j=1(\mathrm{LH})\end{array}$ \\
\hline $\begin{array}{c}i=3 \\
j=2(\mathrm{HL})\end{array}$ & $\begin{array}{c}i=4 \\
j=2(\mathrm{HH})\end{array}$ & $\begin{array}{c}\text { Horizontal orientation } \\
\text { sub image }\end{array}$ \\
\hline \multicolumn{2}{|c|}{$\begin{array}{l}i=6 \\
j=1(\mathrm{HL})\end{array}$} & $\begin{array}{l}i=7 \\
j=1(\mathrm{HH})\end{array}$ \\
\hline $\begin{array}{l}\text { Vertical } \\
\text { sub }\end{array}$ & $\begin{array}{l}\text { orientation } \\
\text { image }\end{array}$ & $\begin{array}{c}\text { Diagonal orientation } \\
\text { sub image }\end{array}$ \\
\hline
\end{tabular}

a)
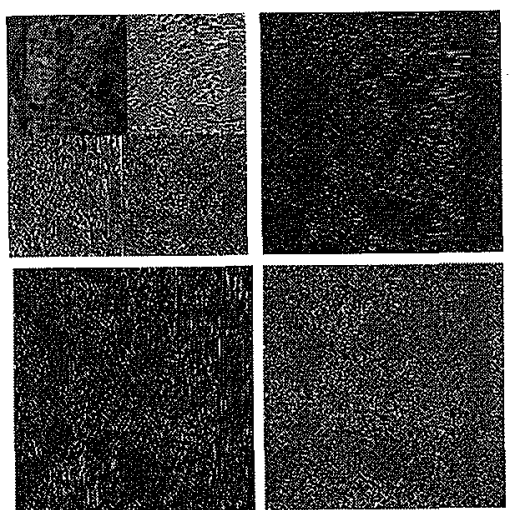

b)

Fig. 1. - a) Separable 2D DWT (1D 8-taps Binomial filters $L$ and $H$ ) image decomposition in 2 resolution levels $j$ and $b$ ) multiresolution (scaled) subimages $i$ of the first type IRLS test image.

IRLS test images:

1. type

2. type

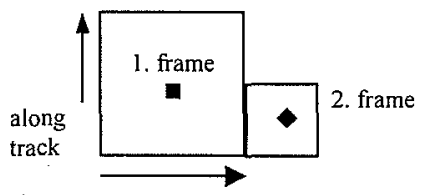

a) along scan

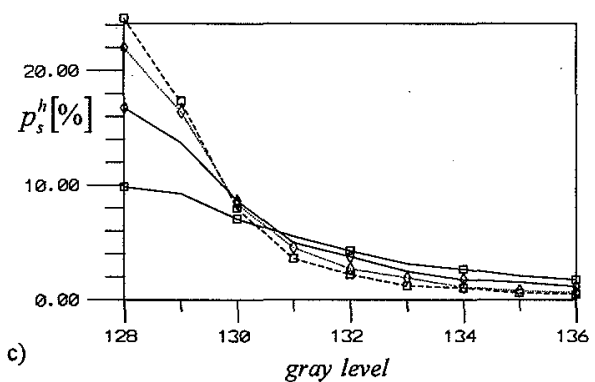

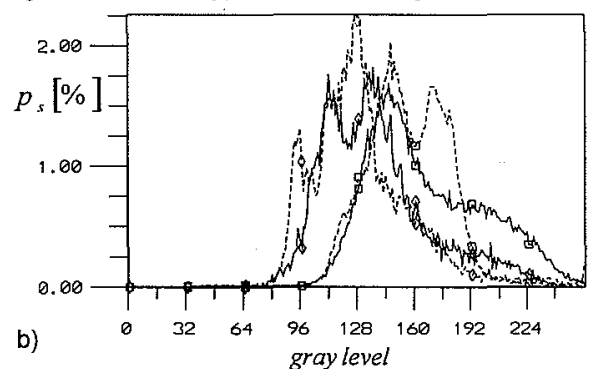

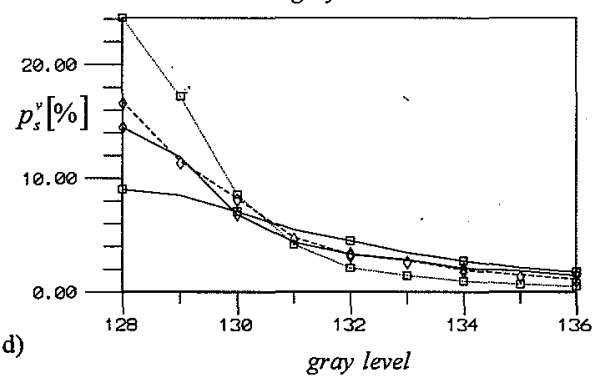


e)

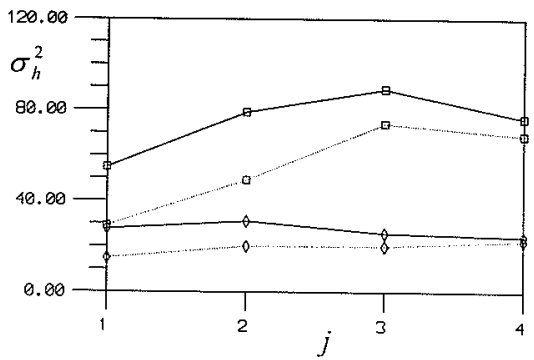

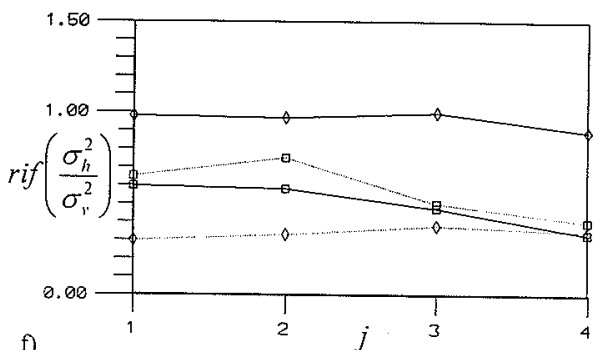

f)

Fig.2. - a) Two analysing segments of IRLS test images and b) their histograms. Histograms of $c$ ) horizontal $p_{s}^{h}(i=5)$ and d) vertical $p_{s}^{\prime \prime}(i=6)$ subimages. e) Variance of horizontal subimages $(i=5)$ and $\left.f\right)$ relative isotropy function rif $\left(\sigma_{h}^{2} / \sigma_{v}^{2}\right)$ vs decomposition level $j$.

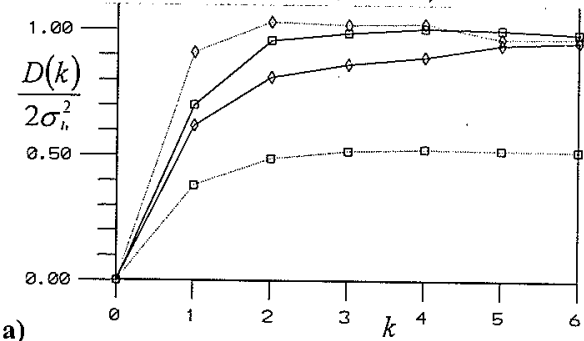

a)

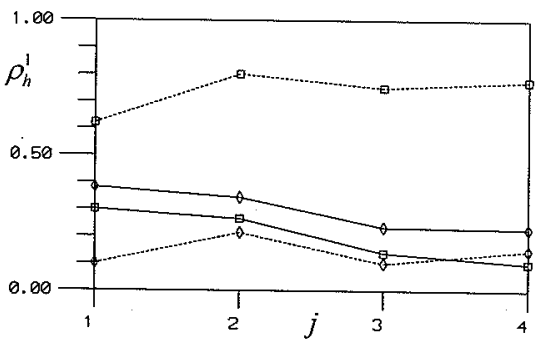

b)
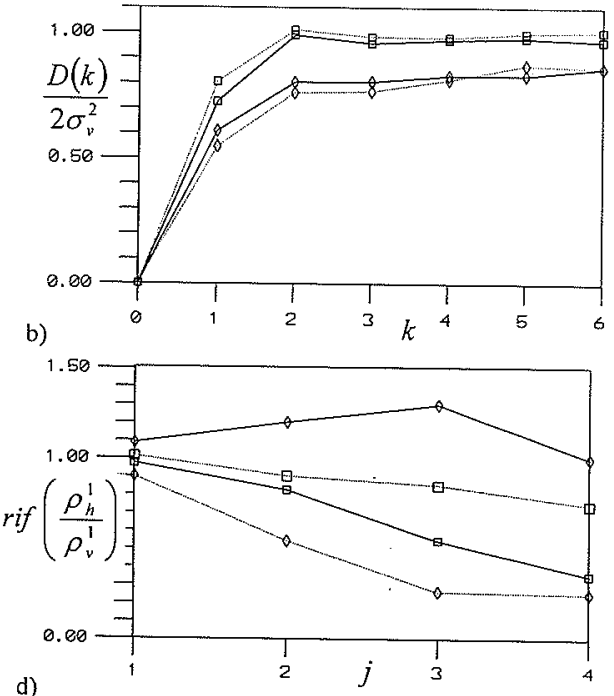

d)

Fig.3. - Structure function $D(k)$ of a) horizontal $(i=5)$ and b) vertical $(i=6)$ subimages. c) Covariance coefficients of horizontal subimages $(i=5)$ and $d)$ relative isotropy function $r i f\left(\rho_{h} / \rho_{\nu}\right)$ vs decomposition level $j$
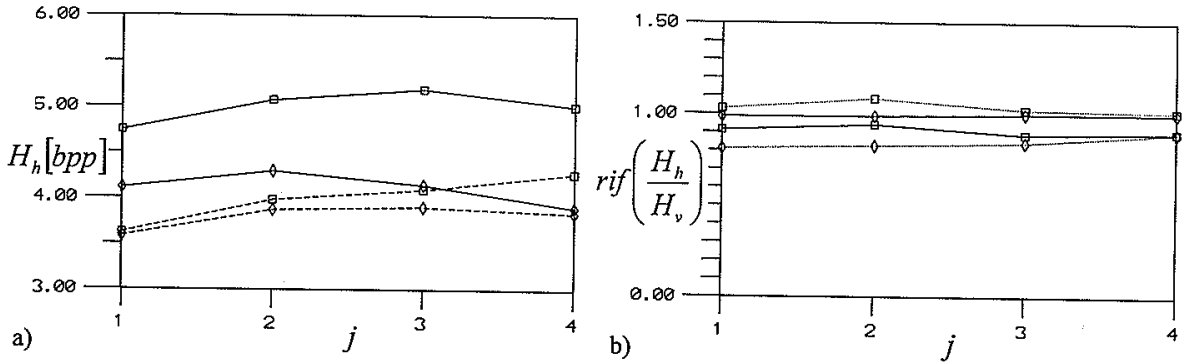

Fig.4. - First order entropy $H$ of a) horizontal $(i=5)$ subimages and b) relative isotropy function rif $\left(H_{h} / H_{v}\right)$ vs decomposition level $j$. 